\title{
Trap1a is an X-linked and cell-intrinsic regulator of thymocyte development
}

\author{
Chi-Shan Li ${ }^{1,5}$, Fei Tang ${ }^{2,5}$, Peng Zhang ${ }^{3}$, Taijiao Jiang ${ }^{3}$, Thomas L Saunders ${ }^{4}$, Pan Zheng ${ }^{2}$ \\ and Yang $\mathrm{Liu}^{2}$
}

The X-linked Trap1a gene encodes the tumor rejection antigen P1A, which is expressed in fetal tissues and multiple lineages of tumor cells. The function of this gene remains unknown. Using chimeric mice with wild-type (WT) and Trap1a-/y bone marrow, we show that Trap1a-ly donor cells are capable of generating most lineages of hematopoietic cells, with the notable exception of T cells. Deletion of Trap1a selectively arrests T-cell development at double-negative stage 1 (DN1, with a CD4-CD8 ${ }^{-}$CD25-CD44 ${ }^{+}$phenotype). Because Trap1a is expressed in $\mathrm{Lin}^{-} \mathrm{Sca}-1^{+} \mathrm{C}-\mathrm{Kit}^{+}$and common lymphoid progenitors but not in immature thymocytes (DN1-DN4), Trap1a mutations affect the differentiation potential of progenitor cells without directly acting on T cells. Despite a similarity in the blockade of DN1 to DN2 transition, the Trap1a-/y DN1 cells have normal expression of c-Kit, in contrast to what was reported in the Notch1-/- DN1. Complementary DNA profiling of Trap1a-ly and WT embryonic stem cells shows that Trap1a does not regulate the Notch pathway. Our data reveal that Trap1a is an X-linked regulator that affects the differentiation potential of progenitor cells into T cells through a Notch-independent mechanism and identify an important function for the Trapla gene.

Cellular \& Molecular Immunology (2017) 14, 685-692; doi:10.1038/cmi.2015.108; published online 11 April 2016

Keywords: Notch; tumor antigens; T cell development

\section{INTRODUCTION}

Thymopoiesis consists of both the orderly differentiation and rapid expansion of T-cell progenitors arriving from peripheral blood. ${ }^{1}$ The early differentiation of immature thymocytes can be marked by the expression of CD44 and CD25 among the $\mathrm{CD}^{-}{ }^{-} \mathrm{CD} 4^{-} \mathrm{CD}^{-}$thymocytes. $^{2}$ Recent studies suggest that a subset of blood $\mathrm{Lin}^{-} \mathrm{Scal}^{+} \mathrm{c}-\mathrm{Kit}^{+}$cells (LSK) are likely the precursors of thymocytes. In the thymus, the LSK are part of the double-negative stage 1 (DN1) $\left(\mathrm{CD} 44^{+} \mathrm{CD} 25^{-}\right)$subset. $^{1}$ Notch/RBP-J signaling is responsible for commitment of progenitors to T-cell lineage (early $\mathrm{T}$-cell progenitor) and the transitions of early T-cell progenitorsfrom DN1 to DN2 $\left(\mathrm{CD} 44^{+} \mathrm{CD} 25^{+}\right)$and from $\mathrm{DN} 3\left(\mathrm{CD} 44^{-} \mathrm{CD} 25^{+}\right)$to $\mathrm{DN} 4$ $\left(\mathrm{CD} 44^{-} \mathrm{CD} 25^{-}\right) .^{3}$ Notch/RBP-J signaling also controls the massive expansion of immature thymocytes. ${ }^{4}$

The X-linked Trapla gene encodes the first known tumor antigen (P1A) recognized by cytotoxic $\mathrm{T}$ cells in mice $^{5}$ and is overexpressed in multiple lineages of tumor cells. ${ }^{6} \mathrm{P} 1 \mathrm{~A}$ is known to be overexpressed in the testis, placenta ${ }^{7,8}$ and embryonic stem (ES) cells (this study). We reported that the transgenic overexpression of Trapla is sufficient to cause thymic lymphoma in an immune-deficient host. ${ }^{9}$ However, the physiological function of P1A remains largely unknown due to the lack of genetic models. To address this issue, we produced ES cells with a floxed Trap1a locus. Because these ES cells were not germline-transmitted, we analyzed the contribution of these mutant ES cells to various tissues in chimeras. Using both blastocyst and bone marrow (BM) chimeras, we observed an unexpected cell-autonomous function of P1A in early thymocyte differentiation, specifically in the DN1 to DN2 transition. Our data reveal an X-linked regulator of the DN1 to DN2 transition in thymocyte development.

\section{MATERIALS AND METHODS}

Generation of Trapla $a^{-l y}$ mutant ES cells

A mouse Trapla genomic clone was obtained by screening a 129/SvJ BAC library. A neomycin/TK cassette flanked by two

\footnotetext{
${ }^{1}$ Institute of Biomedical Sciences, Academia Sinica, Taipei, Taiwan, China; ${ }^{2}$ Center for Cancer and Immunology Research, Children's National Medical Center, Washington, DC, USA; ${ }^{3}$ Institute of Biophysics, Chinese Academy of Science, Beijing, China and ${ }^{4}$ Department of Internal Medicine, School of Medicine, University of Michigan, Ann Arbor, MI, USA

5These authors contributed equally to this work.

Correspondence: Professor P Zheng or Professor Y Liu, Center for Cancer and Immunology Research, Children's National Medical Center, Washington, DC, USA. E-mail: pzheng@cnmc.org or yaliu@cnmc.org

Received: 12 October 2015; Revised: 25 November 2015; Accepted: 25 November 2015
} 
LoxP sites was cloned between Exon 1 and Exon 2, introducing a new BamHI site for genotyping purposes. A third LoxP site was located before Exon 1. This construct was electroporated into murine male X:Y R1 ES cells, ${ }^{10}$ and homologous recombination was confirmed by Southern blot analysis after BamHI digestion. Positive ES cell clones were then electroporated with a Cre vector to eliminate the neomycin/ TK cassette and Trapla Exon 1 (Figure 1a). ES cells were co-cultured with mouse embryonic fibroblast feeder cells in Dulbecco's modified Eagle's medium containing 15\% fetal calf serum, $0.1 \mathrm{~mm} \beta$-mercaptoethanol, $10^{3} \mathrm{u} / \mathrm{ml}$ leukemia inhibitory factor and $4 \mathrm{~mm}$ glutamine as previously described. ${ }^{11}$

\section{Contribution of mutant ES cells to different organs}

Trap1 $a^{-l y}$ ES cells (CD45.2) were microinjected into B6.SJLPtprcaPep3b/BoyJ blastocysts (CD45.1) by the Transgenic Animal Model Core at the University of Michigan. The resulting pups were assessed initially for chimerism based on coat coloration. Genomic DNA was isolated from tissues of chimeric mice using a
DNeasy Blood \& Tissue Kit (Qiagen, Valencia, CA, USA). ES cell contribution was initially tested by quantitative PCR. The contribution of Trapla $1 a^{-1 y}$ ES cells to various tissues of chimeric mice was determined by using primers specific for the LoxP site. The amount of Trapla Exon 2 was used as an internal control. The contribution ratio of Trap $1 a^{-1 y}$ ES cells in different organs was calculated as 2 to the power of the Ct value of the specific LoxP site subtracted from the corresponding $\mathrm{Ct}$ value of the P1A Exon 2. This value was normalized against the value from Trap $1 a^{-1 y} \mathrm{ES}$ cells, which is defined as $100 \%$. The following primers were used for detecting the LoxP site: forward, $5^{\prime}$-CCCTAATGTATGCTATA CGAAGTTAT-3'; reverse, 5'-GGGGTCCTGTAAAGGAAAGG-3' The following primers were used for Trapla Exon 2: forward, 5'-TCCACGACCCTAATTTCC-3'; reverse, 5'-GCATGCCTAA GGTGAGAAGC-3'.

\section{Reverse transcription (RT)-PCR}

Total RNA was isolated from Trap $1 a^{+/ y}$ and Trap $1 a^{-/ y}$ ES cells using a RNeasy Mini Kit (Qiagen). Complementary DNA was a

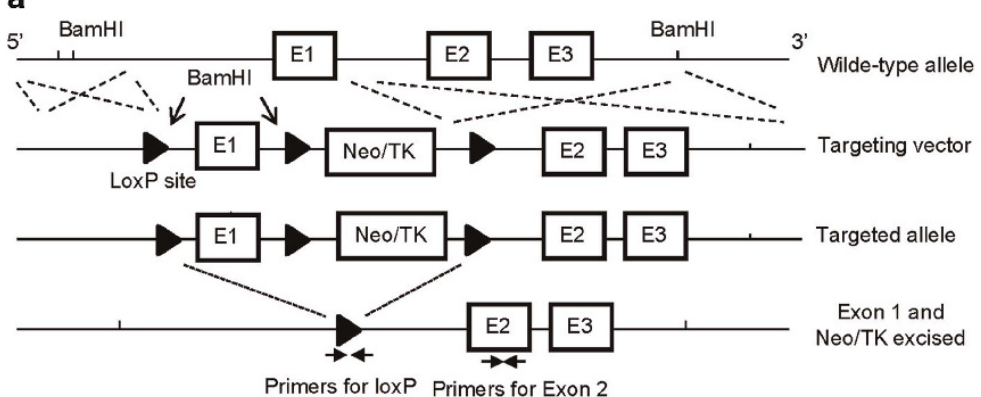

b

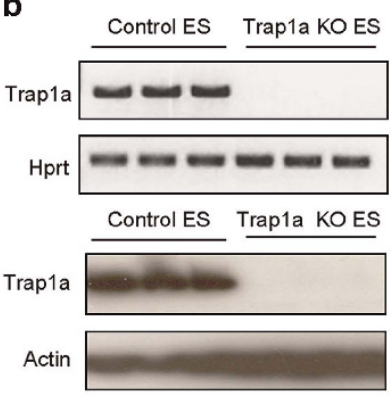

C

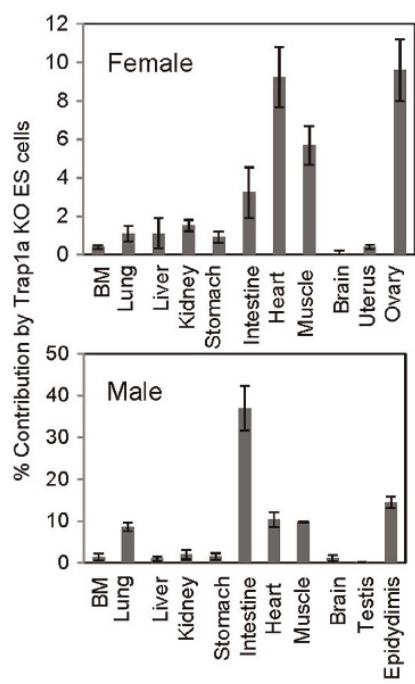

d
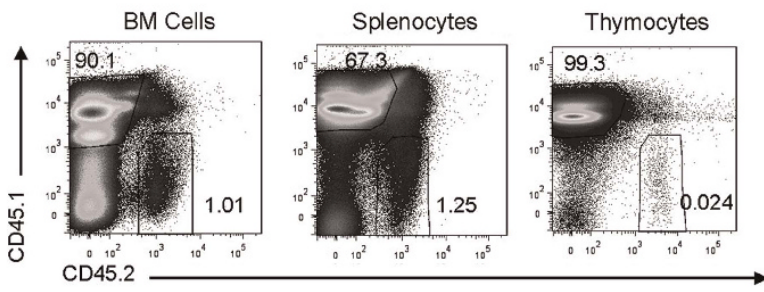

e

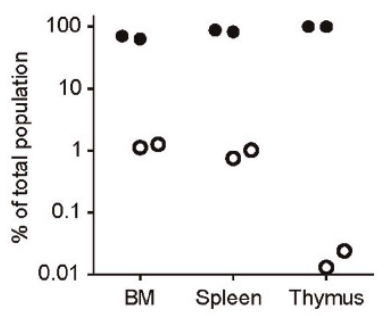

Figure 1 Contribution of Trapla-ly ES cells to mouse tissues through blastocyst chimera analysis. (a) Diagram of constructs and mutant alleles. (b) Confirmation of Trapla inactivation in ES cell clones by RT-PCR and western blot analysis. The Trapla fl/y ES cells were electroporated with a vector expressing Cre recombinase. Three clones in control and Cre-transduced groups were compared for RNA and protein expression. (c) Contribution of Trapla-ly ES cells to various tissues and organs. Chimera mice with greater than $50 \%$ contribution based on coat color were analyzed for contribution of Trapla ${ }^{-l y}$ cells based on quantitative PCR. (d) Flow cytometric quantitation of Trapla-ly $\left(\mathrm{CD} 45.2^{+}\right)$hematopoietic cells in the BM, spleen and thymus. Data from the female chimera mouse are presented; similar results were obtained in the male chimera. (e) \% Contribution of CD $45.2^{+}$cells in the male and female chimera mice. $\mathrm{BM}$, bone marrow; ES cells, embryonic stem cells; RT-PCR, reverse transcriptase-polymerase chain reaction. 


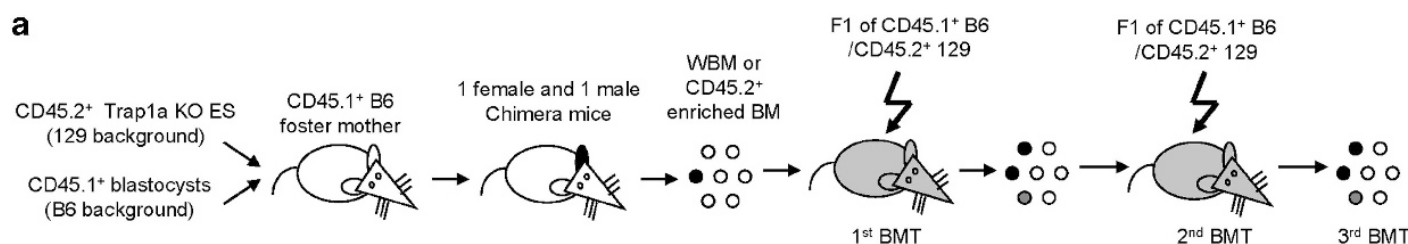

b
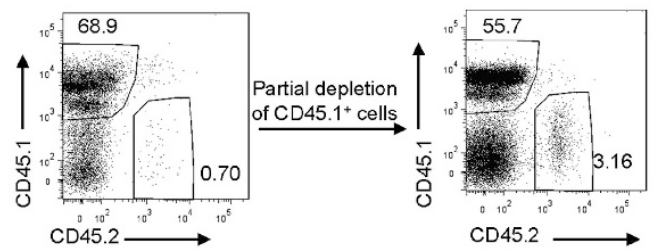

c

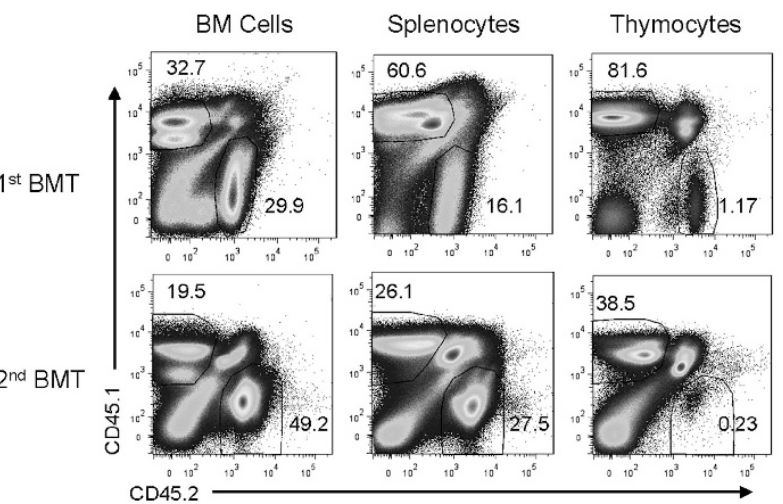

d

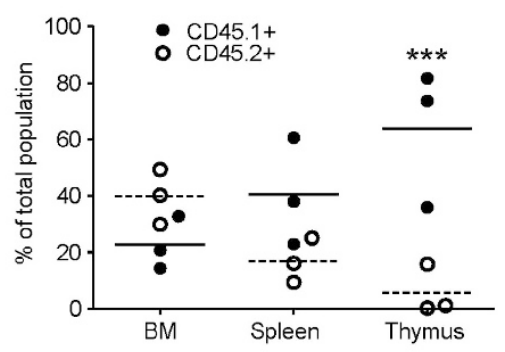

e

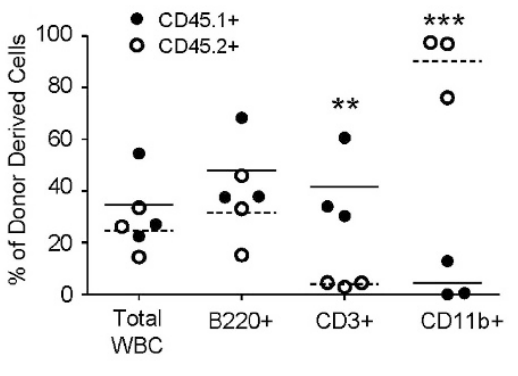

Figure 2 Cell-autonomous and long-lasting effect of Trapla mutation on thymocyte production. (a) Diagram of experimental design. (b) Evidence of partial enrichment of mutant bone marrow cells by negative selection with anti-CD45.1 beads. (c) Impact of Trap1a mutation on thymocyte production in serial transplantation. Data shown are representative profiles of first and second transplantation. (d) Selective reduction of thymocyte production after Trapla deletion. Data shown are \% of WT and Trapla-/y leukocytes at 4 or 11 weeks after second transplantation. (e) Trapla deletion causes selective reduction of T cells and increase of myeloid cells in the peripheral blood at 11 weeks after second transplantation. ${ }^{* *} P<0.01 ;{ }^{* *} P<0.001$. WT, wild type.

synthesized using a SuperScript first-strand synthesis system kit (Invitrogen). For quantitative PCR, the expression of Trapla was amplified by the forward primer 5 '-AGAGATGAGCGTGG AAATGG-3' and the reverse primer $5^{\prime}$-CAGGAAATTAGGGT CGTGGA-3'. The Hprt gene was used as an internal control, and the primers were 5'-CAGGCCAGACTTTGTTGGAT-3' (forward) and 5'-GCGCTCATCTTAGGCTTTGT-3' (reverse). For conventional PCR, primers specific for Trapla (forward: 5'-ATCTAGGGTGGCTGGTCTTC-3'; reverse: 5'-CCATTTCC TCTTCCTCCTCC-3') were used to validate its expression in the mouse LSK population.

\section{Western blot analysis}

Samples were lysed in protein lysis buffer $(50 \mathrm{~mm}$ Tris- $\mathrm{HCl}$, $\mathrm{pH} 7.4,150 \mathrm{~mm} \mathrm{NaCl}, 0.5 \% \mathrm{NP}-40$ ), and protease inhibitor cocktails (Sigma, St Louis, MO, USA) including 4-(2-aminoethyl) benzenesulfonyl fluoride hydrochloride, Aprotinin, Bestatin, E-64, Leupeptin and Pepstatin A were added. Cell lysates were prepared and $50 \mu \mathrm{g}$ of protein per lane was loaded and separated by $10 \%$ sodium dodecyl sulfate-polyacrylamide gel electrophoresis, transferred to polyvinylidene fluoride membranes, and incubated with corresponding antibodies, including rabbit anti-P1A antibody $^{9}$ (1:500 dilution), anti-actin mouse antibody (Sigma, clone AC-15, 1:5000 dilution). Anti-rabbit or anti-mouse IgG horseradish peroxidase-linked antibody at 1:3500 dilution (GE Healthcare, Little Chalfont, UK) was used as a secondary antibody. Antibodies were detected with a chemiluminescence reaction using an enhanced chemiluminescence kit (Amersham Biosciences, Little Chalfont, UK) and visualized with exposure to film.

\section{BM transplantation}

Because Trap $1 a^{-l y}$ ES only contributed $\sim 1 \%$ to bone marrow cells in the chimeric mice, some experiments used anti-fluorescein isothiocyanate microbeads (Miltenyi Biotec, San Diego, CA, USA) to partially delete fluorescein isothiocyanate-labeled CD $45.1^{+}$cells

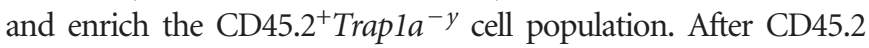
enrichment, or without enrichment, a total of $5-7 \times 10^{6}$ chimeric BM cells were injected intravenously into lethally irradiated (129/ $\mathrm{Sv} \times \mathrm{C} 57 \mathrm{BL} / 6)$ F1 mice $\left(\mathrm{CD} 45.1^{+} \mathrm{CD} 45.2^{+}\right)$by a ${ }^{137} \mathrm{Cs} \gamma$-ray source delivering a total of 1100 rads. All animal experiments were conducted in accordance with accepted standards of animal care and approved by the Institutional Animal Care and Use Committee of the University of Michigan and the Children's National Medical Center Research Institute. 

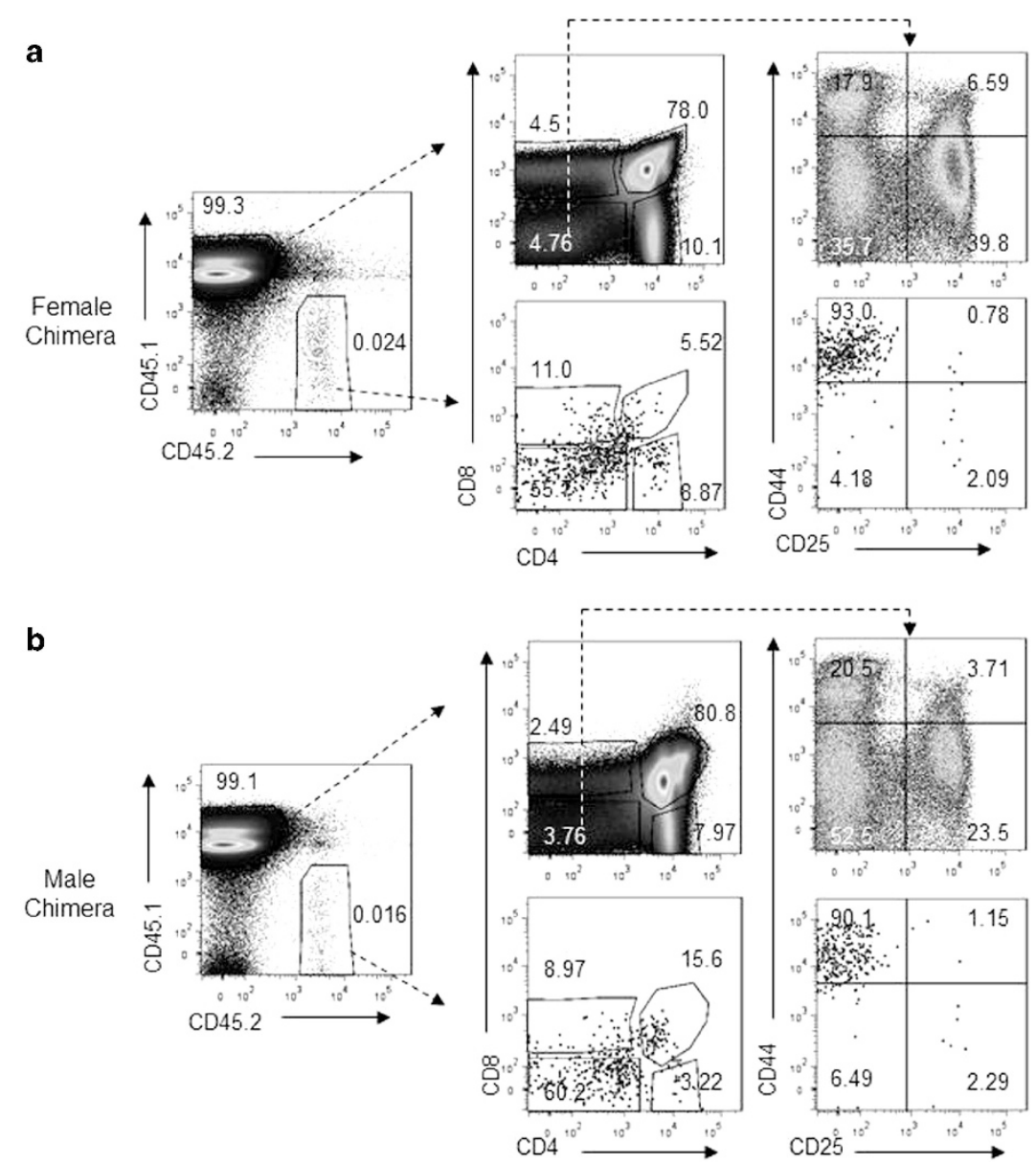

Figure 3 Selective blockade of DN1-DN2 transition by Trap1a deletion as revealed by flow cytometry. Data shown are profiles of thymocytes from the female (a) and male (b) blastocyst chimera mice.

Flow cytometric analysis and cell sorting

Peripheral blood was collected from the orbital sinus of mice. BM cells were collected from the tibia and femur of hind legs. Spleen and thymus were homogenized and passed through a cell strainer to generate a single-cell suspension. Cells were stained in phosphate-buffered saline plus $2 \%$ fetal bovine serum for $20 \mathrm{~min}$ on ice with fluorochrome-conjugated monoclonal antibodies (mAbs) at 1:200 or 1:100 dilution. The following mAbs from BD Biosciences (San Diego, CA, USA), eBioscience (San Diego, CA, USA) or BioLegend (San Diego, CA, USA) were used: anti-CD45.1 (A20), anti-CD45.2 (104), anti-CD3 (145-2C11), anti-CD4 (GK1.5), anti-CD8 (53-6.7), anti-CD25 (PC61.5), antiCD44 (IM7), anti-CD11b (M1/70), anti-B220 (RA3-6B2), antiTER-119 (TER-119), anti-Gr-1 (RB6-8C5), anti-Sca-1 (D7) and anti-c-Kit (2B8). After surface staining, cells were subsequently analyzed with a BD LSR II flow cytometer. The data were analyzed using FlowJo software (Tree Star, San Diego, CA, USA). Cell sorting was performed using a FACSAria (BD Biosciences).

\section{cDNA microarray analysis}

Gene expression profiling analysis of Trap $1 a^{+/ y}$ and the Trap1 $a^{-/ y}$ ES cells was carried out using an Affymetrix GeneChip Mouse Genome 4302.0 array. The RNA samples were sent to the
Affymetrix (Cleveland, OH, USA) and Microarray Core Facility at the University of Michigan for full service sample preparation. Probe intensities were measured and then processed with Affymetrix GeneChip operating software into image analysis (CEL) files. The Affymetrix CEL files were normalized using the Robust Multi-array Averaging method ${ }^{12}$ and affy. ${ }^{13}$ Then, we used limma ${ }^{14}$ to identify differentially expressed genes. Functional analysis (gene ontology enrichment) of differentially expressed genes was assessed using the DAVID database (http://david.abcc.ncifcrf.gov/).

\section{In silico data analysis}

The RNA-seq raw data of thymocytes (double negative (DN) cells, double positive cells and natural regulatory $\mathrm{T}$ cells) were accessed through GEO Series accession number GSE48138. ${ }^{15}$ Alignment of sequencing reads to the reference genome (version mm10) was performed using TopHat. ${ }^{16}$ Then, the gene expression level was measured by RPKM and calculated by DEGseq. ${ }^{17}$ The expression values of hematopoietic stem cells, LSK and common lymphoid progenitors (CLP) were extracted from the expression result file through GEO Series accession numbers GSE50896 ${ }^{18}$ and GSE50739. ${ }^{19}$ 

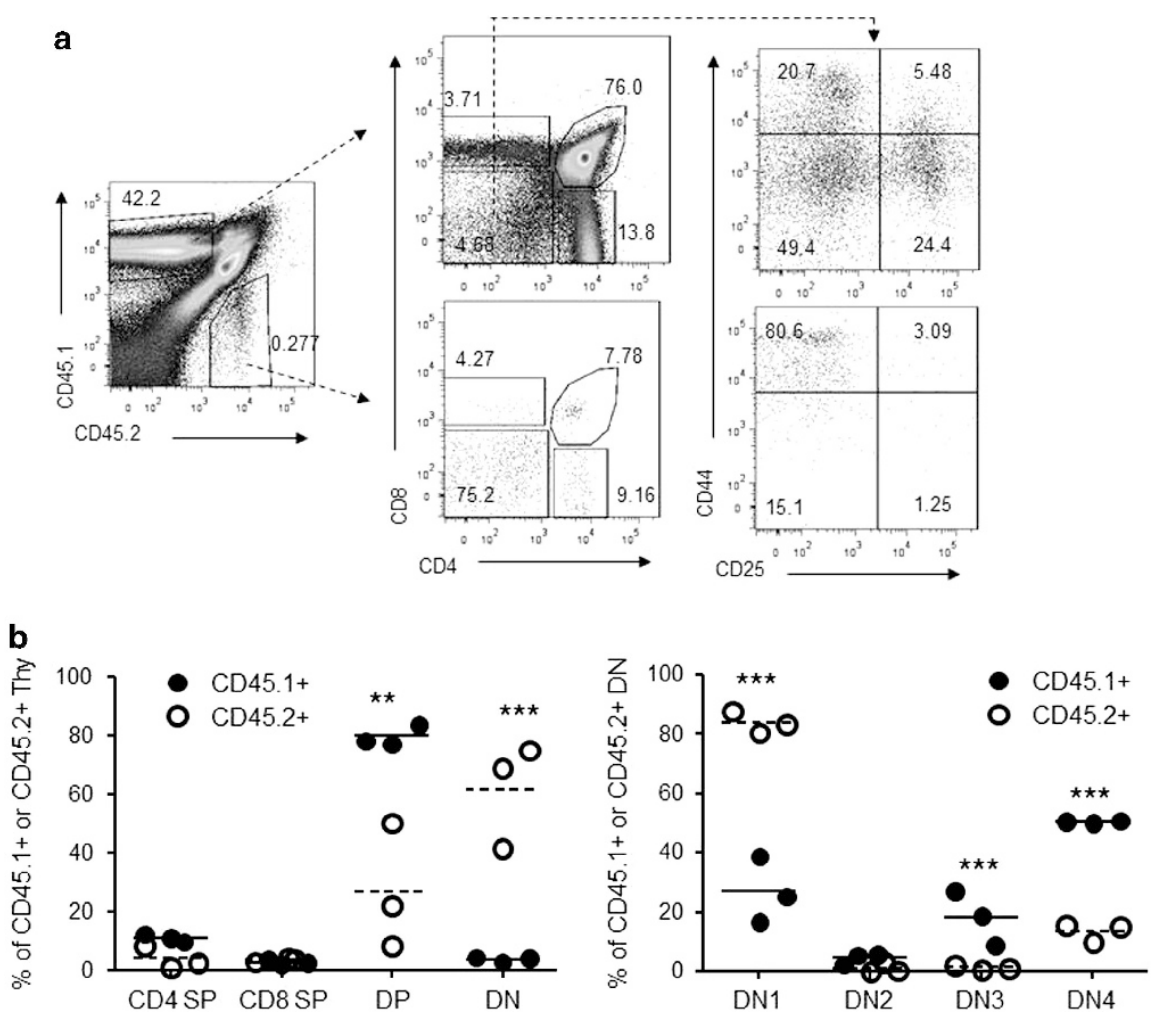

Figure 4 Cell-intrinsic Trapla mutation is responsible for blockade of DN1-DN2 transition. (a) Representative profile of bone marrow chimera mouse at 11 weeks after the second round of transplantation. (b) Accumulation of DN1 cells in Trapla-ly thymocytes. The thymocytes were harvested respectively at 11 or 12 weeks after the second round of transplantation or 7 months after the first round of transplantation.

\section{Statistics}

Data (shown as bar graphs) are presented as the mean \pm s.e.m. (Figure 1c) or mean \pm s.d. (Figure 5e). Two-tailed Student $t$-tests were used for comparison between the two experimental groups. Solid lines and dotted lines in the scatter plots represent mean values for the indicated groups. Statistical significance was determined as ${ }^{\star} P<0.05,{ }^{\star *} P<0.01$ and ${ }^{\star *}{ }^{\star} P<0.001$.

\section{RESULTS}

\section{Chimera analysis revealed differential contributions of} Trap1a-deficient ES cells to different organs

To understand the function of Trap1a, we generated ES cell lines containing a Trap1a triple LoxP gene-targeting vector that included a selectable neomycin/TK cassette and LoxP-flanked Trapla Exon 1 in the mouse X chromosome (Figure 1a). ES clones devoid of P1A protein were obtained by electroporation with a Cre recombinase expression vector (Figure 1b). Unfortunately, the ES cells did not give germline transmission even before Trapla deletion despite repeated attempts, perhaps because the neomycin/TK cassette may have subtly affected Trap1a expression in the hemizygous male-derived ES cells.

As an alternative approach, we removed the neomycin/TK cassette and the Trap1a Exon 1 by electroporation with a Cre recombinase expression vector into ES cells. Cre excised the intervening DNA segment and resulted in a single remaining LoxP site (Figure 1a). Several single Trap1a-null clones were selected and cultured for further analysis. As shown in
Figure $1 \mathrm{~b}$ by RT-PCR and western blotting, we successfully knocked out Trap1a expression in ES cells after removing Trap1a Exon 1. This deletion did not affect ES cell colony morphology or growth rate (Supplementary Figure S1). Moreover, the mutant ES cells formed teratomas of comparable sizes to those derived from WT ES cells. Moreover, histological analyses demonstrated that the mutant ES cells were capable of forming mesoderm, endoderm, ectoderm and germ cells (Supplementary Figure S2).

To determine whether Trap $1 a^{-/ y}$ ES cells could contribute to different organs, we injected them (derived from 129 ES cell line R1, CD45.2) into C57BL/6 (CD45.1) WT blastocysts. We characterized two chimeras with greater than $50 \%$ coat color contribution from Trap $1 a^{-/ y}$ ES cells. Through qPCR, the contribution ratio of Trapla $a^{-/ y}$ ES cells in different organs was calculated as 2 to the power of the Ct value of the specific LoxP site subtracted from the corresponding Ct value of P1A Exon 2 (internal control). This value was normalized against the value from Trap $1 a^{-/ y}$ ES cells, which is defined as $100 \%$. The organs and tissues that we analyzed included the lung, liver, kidney, stomach, intestine, heart, muscle, brain, uterus, ovary, testis, epididymis and BM. The primer positions are depicted in the upper panel of Figure 1c. As shown in the lower panel of Figure 1c, the Trap1a $1 a^{-/ y}$ ES cells contributed to various internal organs, although the contribution ratios differed among organs. Consistent with its abundant expression in the 
a

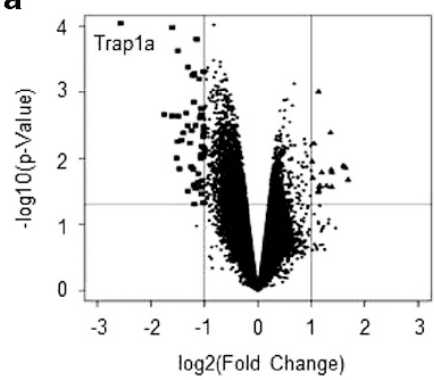

b

\begin{tabular}{cccc}
\hline Term & Gene_Count & $\%$ & p-Value \\
\hline developmental process & 17 & 27.9 & $2.70 \mathrm{E}-03$ \\
cellular developmental process & 12 & 19.7 & $5.40 \mathrm{E}-03$ \\
multicellular organismal development & 15 & 24.6 & $8.60 \mathrm{E}-03$ \\
cell differentiation & 11 & 18 & $1.20 \mathrm{E}-02$ \\
regulation of metabolic process & 16 & 26.2 & $1.40 \mathrm{E}-02$ \\
anatomical structure development & 13 & 21.3 & $1.70 \mathrm{E}-02$ \\
regulation of cellular metabolic process & 15 & 24.6 & $2.00 \mathrm{E}-02$ \\
\hline
\end{tabular}

c

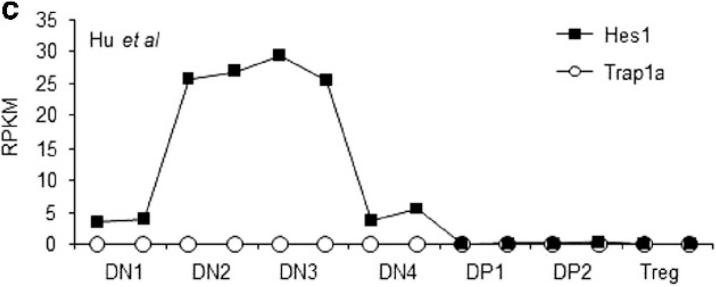

d HSC LSK
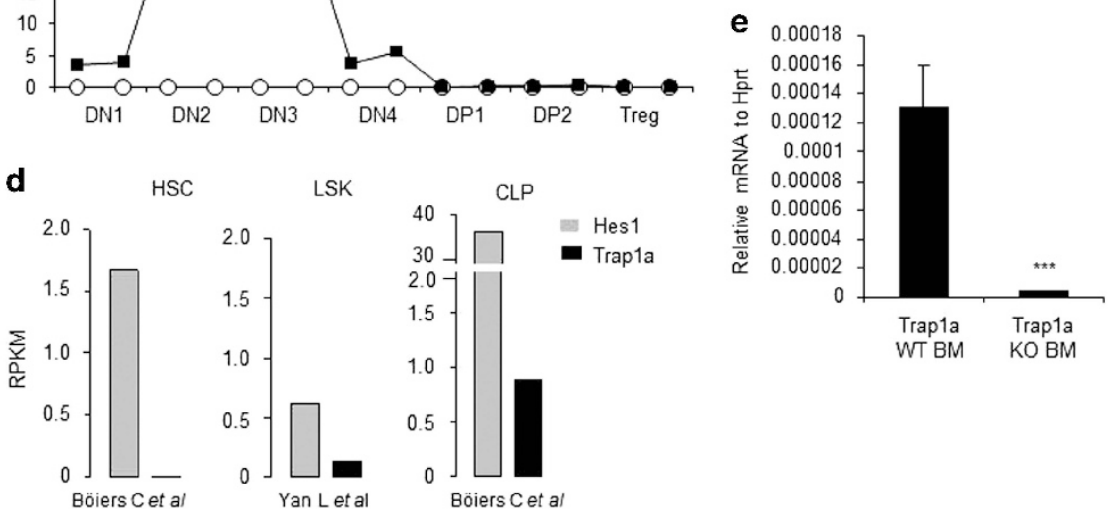

f

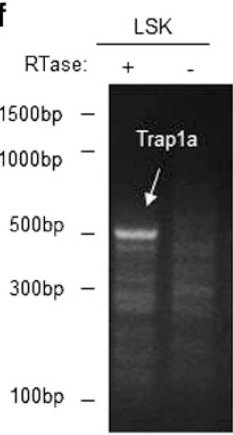

Figure 5 Function of Trapla gene based on global expression analysis. (a) Identification of genes that are affected by Trap1a deletion in ES cells. The $X$-axis shows the ratios of expression levels between WT and Trapla-ly ES cells, while the Y-axis shows -log10 $P$ values. The large symbols indicate genes that are either upregulated (triangles) or downregulated (squares) by Trapla deletion. (b) Functional enrichment analysis suggests that Trapla may be involved in cellular development and metabolic pathways. (c-e) Trapla is expressed in LSK and CLP but not in thymocytes and hematopoietic stem cells. (c, d) In silico analyses of RNAseq data from previous publications. (e) GPCR analysis of bone, marrow cells sorted from bone marrow chimera mice. (f) RT-PCR verification of Trapla expression in the sorted LSK from CD45.1 (WT) and CD45.2 (mutant) LSK. CLP, common lymphoid progenitor; LSK, Lin ${ }^{-} / \mathrm{SCA}^{+} / \mathrm{Kit}^{+}$; qPCR, quantitative polymerase chain reaction; RT-PCR, reverse transcriptase-polymerase chain reaction.

testis, ${ }^{20}$ Trap1a-deficient ES cells failed to contribute to testis tissues.

Using the CD45 congenic markers, we quantified the relative number of Trap $1 a^{-/ y}$ ES cell-derived cells in major hematopoietic tissues, including the BM, spleen and thymus. As shown in Figures $1 \mathrm{~d}$ and e, the number of CD45.2 cells in the spleen and BM was $\sim 1 \%$. Surprisingly, the contribution to thymocytes was further reduced by 40 -fold, to $0.024 \%$. These data suggest that Trap1a may affect thymocyte development.

Cell-intrinsic function of Trap1a in thymocyte development To determine whether the Trapla gene function in hematopoiesis is cell-intrinsic, we transplanted BM cells from mouse chimeras (either CD $45.1^{+}$or CD $45.2^{+}$) into lethally irradiated $(129 / \mathrm{Sv} \times \mathrm{C} 57 \mathrm{BL} / 6) \mathrm{F} 1$ mice $\left(\mathrm{CD} 45.1^{+} \mathrm{CD} 45.2^{+}\right)$(Figure $\left.2 \mathrm{a}\right)$. In this setting, the WT cells were CD $45.1^{+} \mathrm{CD} 45.2^{-}$, the Trap $1 a^{-l y}$ cells were $\mathrm{CD} 45.1^{-} \mathrm{CD} 45.2^{+}$, whereas the residual recipient leukocytes were $\mathrm{CD} 45.1^{+} \mathrm{CD} 45.2^{+}$(Figure 2a). Because the number of CD $45.2^{+} \mathrm{BM}$ cells in the female chimera was exceedingly low, we partially enriched CD $45.1^{-} \mathrm{CD} 45.2^{+}$cells by using negative selection (Figure $2 \mathrm{~b}$ ). This partial enrichment is sufficient because the proportion of stem/progenitor cells is higher among the mutant hematopoietic cells (data not shown). For comparison, male BM cells were directly used without enrichment. A similar trend was found with or without enrichment. We present data mainly from the female chimera because the higher proportion of mutant-derived cells in the female made the quantitation more reliable. As shown in Figure 2c, with the partial enrichment of CD45.1- CD45.2 ${ }^{+}$ donor cells, the proportions of mutant CD45.1- CD45.2 $2^{+}$cells were comparable to WT CD $45.1^{+} \mathrm{CD} 45.2^{-}$donor cells in the $\mathrm{BM}$ and spleen. However, the thymus was largely depleted of CD45.1 ${ }^{-}$CD 45.2 $2^{+}$cells (Figure 2c). The defects were even more striking in repeated transplantation (Figures $2 \mathrm{c}$ and $\mathrm{d}$ ). The marked and selective reduction of thymocytes from the $\mathrm{CD} 45.1^{-} \mathrm{CD} 45.2^{+}$donor, as summarized in Figure $2 \mathrm{~d}$, demonstrated a critical cell-intrinsic function of Trap1a in T-cell development. Consistent with a defective T-cell development, the proportion of $\mathrm{T}$ cells in the peripheral blood was also reduced, whereas that of myeloid cells was increased (Figure 2e). 

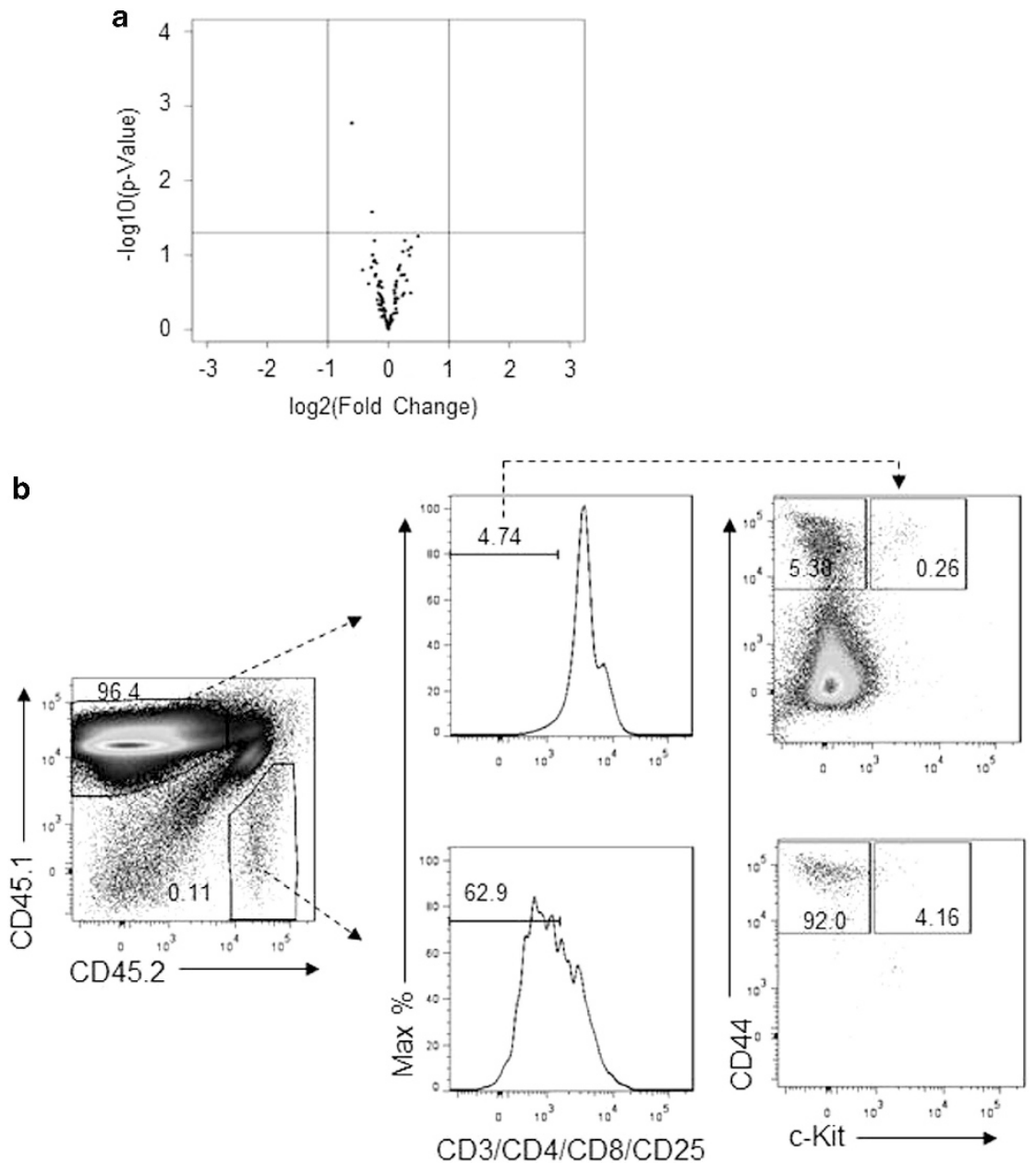

Figure 6 Trapla mutation does not affect Notch signaling. (a) None of the 43 Notch signaling genes are affected by more than twofold. The X-axis shows the ratios of expression levels between WT and Trapla-ly ES cells, while the Y-axis shows -log10 $P$ values. (b) Distribution of c-Kit expression among the DN1 thymocytes from WT and Trapla ${ }^{-l y}$ stem cells. Data shown are representative profiles of thymocytes based on CD3/CD4/CD8/CD25/CD44 and c-Kit markers and have been reproduced in three independent experiments. ES cells, embryonic stem cells; WT, wild type.

\section{A critical role for Trap1a in DN1 to DN2 transition}

To understand which developmental stage is regulated by Trap 1a, we further analyzed the thymocyte composition of the blastocyst mouse chimeras based on the expression of CD4, CD8, CD44 and CD25. As shown in Figure 3, although the $\mathrm{CD} 4^{-} \mathrm{CD} 8^{-}$thymocytes were a minority among the WT cells $\left(\mathrm{CD} 45.1^{+}\right)$, they represented the overwhelming majority of the $\mathrm{CD} 45.2$ thymocytes. After the $\mathrm{CD}^{-} \mathrm{CD}^{-}$thymocytes were further subdivided into DN1-DN4 based on the distribution of CD44 and CD25 markers, it was clear that essentially all DN had a phenotype of CD $44^{\text {hi }} \mathrm{CD} 25^{-}$in the CD 45.2 thymocytes. The selective defects in the blastocyst chimera were substantiated in multiple rounds of BM transplantation (Figures $4 \mathrm{a}$ and b). Therefore, Trap1a deletion caused cell-intrinsic defects in DN1-DN2 transition.

Because there were too few Trap $1 a^{-/ y}$ thymocytes for expression profiling, we profiled the Trapla $1 a^{+/ y}$ and the Trap $1 a^{-1 y}$ ES cells and compared the Notch signaling signature. Using twofold changes and a $P$ value of 0.05 as the cutoff, we observed 51 downregulated genes and 17 upregulated genes after Trap1a deletion (Figure 5a and Supplementary Dataset 1). As expected, the most downregulated gene was Trapla. Functional enrichment analysis suggested that these genes affect cellular metabolism and development (Figure 5b).

Theoretically, Trap1a may act directly either to promote the DN1-DN2 transition or to determine the potential of T-cell progenitors. The former predicts the expression of Trapla in the thymocytes, whereas the latter does not have such a requirement. We carried out in silico analyses for Trap1a expression. As shown in Figure $5 c$, RNAseq data ${ }^{15}$ showed that Trap1a was not expressed in thymocytes, whereas databases generated by others ${ }^{18,19}$ revealed low levels of expression in BM LSK stem/progenitor cells and CLP cells (Figure 5d). Because thymocyte progenitors were identified as part of the LSK population, ${ }^{21}$ we used PCR to validate Trapla expression in LSK cells. As shown in Figure 5e, Trapla was detected in the WT but not the CD45.2 $2^{+}$mutant BM cells by qPCR. Further investigation by conventional PCR confirmed the expression of 
Trap1a in sorted LSK cells from the BM of WT mice (Figure 5f). Therefore, the expression pattern suggested that Trap1a affects the potential of $\mathrm{T}$ cell progenitors rather than directly acting on DN1 to promote the DN1 to DN2 transition.

The nearly complete inhibition of the DN1 to DN2 transition is reminiscent of the phenotype of Notch1-/thymocytes. ${ }^{4}$ To determine whether the Notch signaling pathway was affected, we compared the expression of genes involved in Notch pathways in Trap1 $a^{+/ y}$ and Trap1a-ly ES cells. As shown in Figure 6a and Supplementary Dataset 2, none of the genes in the Notch pathway were affected by twofold or more. Because Notch1 deletion eliminated c-Kit expression in DN1 cells, ${ }^{21}$ we compared the expression of c-Kit in DN1 thymocytes. As shown in Figure 6b, the distribution of c-Kit expression in DN1 was unaffected by Trapla deletion. These data suggest that Trapla deletion does not affect thymocyte development through inhibition of Notch signaling.

\section{DISCUSSION}

Taken together, our data revealed Trapla as a new regulator of the DN1-DN2 transition. This checkpoint is distinct from Notch1 signaling at three levels. First, phenotypically, Notch1deficient DN1 thymocytes were blocked prior to the expression of c-Kit, the earliest marker of T-cell progenitors, whereas the Trap1a-deficient thymocytes had normal expression of c-Kit. Second, although Notch signaling is active at the DN1-DN4 based on the expression of its target genes, Trapla is expressed only in progenitor cells before they reach the thymus. Third, gene signature analysis of ES cells suggests that Trapla deletion does not affect the expression of genes involved in Notch signaling. It is intriguing to note that because Trap1a expression cannot be found in developing thymocytes the potential for $\mathrm{T}$-cell fate can be determined before $\mathrm{T}$-cell progenitors reach the thymus.

P1A was the first tumor antigen to be identified. However, no physiological function has been attributed to P1A. By showing a critical role for P1A in T-cell development, our study filled in this major gap. Furthermore, based on the lack of contribution of the Trap $1 a^{-/ y}$ ES cells to several tissues, particularly the uterus, testis and brain, it is likely that P1A plays a fundamental role in the development of vital organs. Additional studies will be needed to determine the molecular mechanism by which P1A controls development of multiple cell types.

\section{CONFLICT OF INTEREST}

The authors declare no conflict of interest.

\section{ACKNOWLEDGEMENTS}

This work is supported by grants from the National Institutes of Health (AI64350, CA171972, CA58033 and AG036690).
1 Schwarz BA, Bhandoola A. Circulating hematopoietic progenitors with T lineage potential. Nat Immunol 2004; 5: 953-960.

2 Ceredig R, Rolink T. A positive look at double-negative thymocytes. Nat Rev Immunol 2002; 2: 888-897.

3 Schmitt TM, Ciofani M, Petrie HT, Zuniga-Pflucker JC. Maintenance of $\mathrm{T}$ cell specification and differentiation requires recurrent notch receptor-ligand interactions. J Exp Med 2004; 200: 469-479.

4 Allman D, Karnell FG, Punt JA, Bakkour S, Xu L, Myung P et al. Separation of Notch1 promoted lineage commitment and expansion/ transformation in developing T cells. J Exp Med 2001; 194: 99-106.

5 Van den Eynde B, Lethe B, Van Pel A, De Plaen E, Boon T. The gene coding for a major tumor rejection antigen of tumor P815 is identical to the normal gene of syngeneic DBA/2 mice. J Exp Med 1991; 173: 1373-1384.

6 Ramarathinam L, Sarma S, Maric M, Zhao M, Yang G, Chen L et al. Multiple lineages of tumors express a common tumor antigen, P1A, but they are not cross-protected. J Immunol 1995; 155: 5323-5329.

7 Boon T, Cerottini JC, Van den Eynde B, van der Bruggen P, Van Pel A. Tumor antigens recognized by $\mathrm{T}$ lymphocytes. Annu Rev Immunol 1994; 12: 337-365.

8 Brandle D, Bilsborough J, Rulicke T, Uyttenhove C, Boon T, Van den Eynde BJ. The shared tumor-specific antigen encoded by mouse gene P1A is a target not only for cytolytic $T$ lymphocytes but also for tumor rejection. Eur J Immunol 1998; 28: 4010-4019.

9 Li CS, Chen C, Zheng P, Liu Y. Transgenic expression of P1A induced thymic tumor: a role for onco-fetal antigens in tumorigenesis. PLOS One 2010; 5: e13439.

10 Nagy A, Rossant J, Nagy R, Abramow-Newerly W, Roder JC. Derivation of completely cell culture-derived mice from early-passage embryonic stem cells. Proc Natl Acad Sci USA 1993; 90: 8424-8428.

11 Hughes ED, Saunders TL. Gene Targeting in Embryonic Stem Cells. Springer-Verlag: Berlin. 2001

12 Irizarry RA, Hobbs B, Collin F, Beazer-Barclay YD, Antonellis KJ, Scherf $U$ et al. Exploration, normalization, and summaries of high density oligonucleotide array probe level data. Biostatistics 2003; 4: 249-264.

13 Gautier L, Cope L, Bolstad BM, Irizarry RA. affy-analysis of Affymetrix GeneChip data at the probe level. Bioinformatics 2004; 20: 307-315.

14 Ritchie ME, Phipson B, Wu D, Hu Y, Law CW, Shi W et al. limma powers differential expression analyses for RNA-sequencing and microarray studies. Nucleic Acids Res 2015; 43: e47.

15 Hu G, Tang Q, Sharma S, Yu F, Escobar TM, Muljo SA et al. Expression and regulation of intergenic long noncoding RNAs during T cell development and differentiation. Nat Immunol 2013; 14: 1190-1198.

16 Kim D, Pertea G, Trapnell C, Pimentel H, Kelley R, Salzberg SL. TopHat2: accurate alignment of transcriptomes in the presence of insertions, deletions and gene fusions. Genome Biol 2013; 14: R36.

17 Wang L, Feng Z, Wang X, Zhang X. DEGseq: an R package for identifying differentially expressed genes from RNA-seq data. Bioinformatics 2010; 26: 136-138.

18 Boiers C, Carrelha J, Lutteropp M, Luc S, Green JC, Azzoni E et al. Lymphomyeloid contribution of an immune-restricted progenitor emerging prior to definitive hematopoietic stem cells. Cell Stem Cell 2013; 13: $535-548$.

19 Yan L, Womack B, Wotton D, Guo Y, Shyr Y, Dave U et al. Tgif1 regulates quiescence and self-renewal of hematopoietic stem cells. Mol Cell Biol 2013; 33: 4824-4833.

20 Uyttenhove C, Godfraind C, Lethe B, Amar-Costesec A, Renauld JC, Gajewski TF et al. The expression of mouse gene P1A in testis does not prevent safe induction of cytolytic T cells against a P1A-encoded tumor antigen. Int J Cancer 1997; 70: 349-356.

21 Tan JB, Visan I, Yuan JS, Guidos CJ. Requirement for Notch1 signals at sequential early stages of intrathymic $\mathrm{T}$ cell development. Nat Immunol 2005; 6: 671-679.

Supplementary Information for this article can be found on the Cellular \& Molecular Immunology website (http://www.nature.com/cmi) 